\title{
Comparative integromics on FAT1, FAT2, FAT3 and FAT4
}

\author{
YURIKO KATOH $^{1}$ and MASARU KATOH ${ }^{2}$ \\ ${ }^{1}$ M\&M Medical BioInformatics, Hongo 113-0033; ${ }^{2}$ Genetics and Cell Biology Section, \\ National Cancer Center Research Institute, Tokyo 104-0045, Japan
}

Received May 2, 2006; Accepted June 5, 2006

\begin{abstract}
WNT5A, WNT5B, WNT11, FZD3, FZD6, VANGL1, VANGL2, DVL1, DVL2, DVL3, PRICKLE1, PRICKLE2, ANKRD6, NKD1, NKD2, DAAM1, DAAM2, CELSR1, CELSR2, CELSR3, ROR1 and ROR2 are planar cell polarity $(\mathrm{PCP})$ signaling molecules implicated in the regulation of cellular polarity, convergent extension, and invasion. FAT1, FAT2, FAT3 and FAT4 are Cadherin superfamily members homologous to Drosophila Fat, functioning as a positive regulator of PCP in the Drosophila wing. Complete coding sequence (CDS) for human FAT1 (NM_005245.3) and FAT2 (NM_001447.1) are available, while artificial CDS for human FAT3 (XM_926199 and XM_936538) and partial CDS for FAT4 (NM_024582.2). Here, complete CDS of human FAT3 and FAT4 were determined by using bioinformatics and human intelligence (Humint). FAT3 gene, consisting of 26 exons, encoded a 4557-aa protein with extracellular 33 Cadherin repeats, one Laminin $\mathrm{G}$ (LamG) domain and two EGF domains. FAT4 gene encoded a 4924-aa protein with extracellular 34 Cadherin repeats, two LamG domains and three EGF domains. Cytoplasmic VCSVxPxLP and SDYXS motifs were identified as novel motifs conserved among FAT1, FAT2 and FAT3 orthologs. Domain architecture comparison and phylogenetic analysis revealed that FAT1, FAT2 and FAR3 were divergent from FAT4. FAT1-MTNR1A locus at $4 \mathrm{q} 35.2$ and FAT3-MTNR1B locus at 11q14.3-q21 were paralogous regions within the human genome. FAT1 mRNA was expressed in embryonic stem (ES) cells, neural tissues, gastric cancer, pancreatic cancer, colorectal cancer, breast cancer, lung cancer and brain tumors. FAT2 mRNA was expressed in infant brain, cerebellum, gastric cancer, pancreatic cancer, ovarian cancer, esophageal cancer, skin squamous cell carcinoma, head and neck cancer. FAT3 mRNA was expressed in ES cells, primitive neuroectoderm, fetal brain, infant brain, adult neural tissues and prostate. FAT4 mRNA was expressed in fetal brain, infant brain, brain
\end{abstract}

Correspondence to: Dr Masaru Katoh, Genetics and Cell Biology Section, National Cancer Center Research Institute, 5-1-1 Tsukiji, Chuo-ku, Tokyo 104-0045, Japan

E-mail: mkatoh@ncc.go.jp; mkatoh@seaple.ne.jp

Key words: bioinformatics, comparative genomics, comparative proteomics, Cadherin, integrome network, systems medicine tumor and colorectal cancer. FAT family members were revealed to be targets of systems medicine in the fields of oncology and neurology.

\section{Introduction}

Drosophila Frizzled, Dishevelled, Diego, Starry night (Flamingo), Van Gogh (Strabismus) and Prickle are core planar cell polarity (PCP) signaling molecules (1-7). Asymmetrical localization of Frizzled - Dishevelled - Diego - Starry night complex and Van Gogh - Prickle complex induces PCP in the Drosophila wing. Human WNT5A, WNT5B, WNT11, FZD3, FZD6, VANGL1, VANGL2, DVL1, DVL2, DVL3, PRICKLE1, PRICKLE2, ANKRD6, NKD1, NKD2, DAAM1, DAAM2, CELSR1, CELSR2, CELSR3, ROR1 and ROR2 are PCP signaling molecules implicated in the regulation of cellular polarity, convergent extension, and invasion (7-26). Activation of PCP signaling pathway controls tissue polarity and cell movement through the activation of RHOA, c-Jun $\mathrm{N}$-terminal kinase (JNK), and nemo-like kinase (NLK) signaling cascades.

Fat, Four-jointed and Dachsous are additional PCP signaling molecules in the Drosophila wing (27-29). Drosophila Fat, functioning as a positive regulator of PCP in the Drosophila wing, is a member of the Cadherin superfamily. Drosophila Fat-like, implicated in the morphogenesis of tubular structures of ectodermal origin, is the homolog of Fat (30). Fat1, Fat2, Fat3 and Fat4 are rodent homologs of Drosophila Fat and Fat-like (31-33). Complete coding sequence (CDS) for human FAT1 (NM_005245.3) and FAT2 (NM_001447.1) are available in the public database, while artificial CDS for human FAT3 (XM_926199 and XM_936538) and partial CDS for FAT4 (NM_024582.2). Here, complete CDS of human FAT3 and FAT4 were determined by using bioinformatics and human intelligence (Humint). Comparative genomics analyses, proteomics analyses and expression profile analyses on the FAT family members were then performed.

\section{Materials and methods}

Determination of complete CDS for FAT3 and FAT4. Human cDNAs, expressed sequence tags (ESTs) and genome sequences, derived from FAT3 and FAT4 transcripts, were searched for with BLAST programs as described previously (34-36). Complete CDS of human FAT3 and FAT4 were determined by assembling exonic regions. 
Comparative genomics analyses. Intra-species comparative genomics analyses were performed as described previously (37-39). Genome sequences corresponding to human FAT1, FAT2, FAT3 and FAT4 genes were searched for with BLAST programs (http://www.ncbi.nlm.nih.gov). TCF/LEF-binding sites within the 5'-flanking promoter region of above genes were searched for based on bioinformatics and manual inspection as described previously (40-42).

Comparative proteomics analyses. Domain architecture analyses of FAT family members were performed by using RPS-BLAST and PSORT II programs. Phylogenetic analysis on FAT family proteins was performed by using the CLUSTALW program. Human FAT1, FAT2, FAT3, rat Fat1, Fat2 and Fat 3 were then aligned by using Genetyx program and manual curation as described previously (43-45).

In silico expression analyses. Expressed sequence tags (ESTs) derived from human FAT1, FAT2, FAT3 and FAT4 genes were searched for by using the BLAST programs as described previously (46-48). The sources of human ESTs derived from $F A T$ family genes were listed up for in silico expression analyses.

\section{Results}

Complete CDS of human FAT3. BLAST programs using rat Fat3 RefSeq (NM_138544.1) revealed that human FAT3 gene was located within AP000722.5, AP000805.4, AP002514.5, AP003718.3, AC067807.10 and AP003171.2 genome sequences. Because the first exon corresponding to the 5'-UTR of human FAT3 mRNA was not detected based on the BLAST programs, we searched for human EST spanning the 5'-UTR to identify BF953408.1 EST. By using the nucleotide sequence of BF953408.1 EST as a query sequence for the BLAST programs, the first exon of human FAT3 gene was identified within AP003124.3 genome sequence.

Exon-intron boundaries of human FAT3 gene were determined based on the consensus sequence of exon-intron junctions. Exon 1 was located within AP003124.3 genome sequence as mentioned above, exons 2 within AP000722.5, exon 3 within AP000805.4, exon 4 within AP002514.5, exons 5-17 within AP003718.3, exons 5-22 within AC067807.10, and exons 22-26 within AP003171.2. Exons 2, 10 and 26 were larger than $3 \mathrm{~kb}$ in length. Human FAT3 gene was found consisting of 26 exons (Fig. 1A).

Because XM_926199 and XM_936538 were human FAT3 predicted sequences with the artificial first exon, complete CDS of human FAT3 was determined by assembling exonic regions (Fig. 1B). Genetyx program revealed that nucleotide position 67-13740 was the coding region. Human FAT3 gene was found to encode a 4557 -amino-acid FAT3 protein (Fig. 1B).

Complete CDS of human FAT4. Preliminary alignment of FAT family members revealed that human FAT4 RefSeq (NM_024582.2) was a partial CDS, lacking N-terminal one thirds of the coding region. BLAST programs using mouse Fat4 RefSeq (NM_183221.2) revealed that human FAT4 gene was located within AC079835.5, AC098865.2 and AC092629.2 genome sequences. The 5'-UTR of FAT family transcripts were interrupted by the first intron; however, putative first exon corresponding to the 5'-UTR of FAT4 gene was not identified in this study due to the absence of EST or cDNA. Complete CDS of human FAT4 was determine by assembling AC079835.5 genome sequence (nucleotide position 102306107480) and NM_024582.2 RefSeq (nucleotide position 70-9669) (Fig. 2A). Genetyx program revealed that nucleotide position 1-14775 was the coding region. FAT4 gene was found to encode a 4924-aa FAT4 protein.

Complete CDS of human FAT2. Although human FAT2 RefSeq (NM_001447.1) spanned the entire coding region, its 5'-end did not extend to the first exon corresponding to 5'-UTR. BLAST programs revealed that DA102144.1 EST spanned to the missing first exon. Therefore, full-length complete CDS of human FAT2 was determined by assembling DA102144.1 EST and NM_001447.1 RefSeq (Fig. 2A).

Comparative genomics analyses on FAT family. FAT1 gene at human chromosome $4 \mathrm{q} 35.2$ was linked to the MTNR1A gene, while FAT3 gene at human chromosome 11q14.3-q21 was linked to the MTNRIB gene (Fig. 2B). Third MTNR1 family gene linked FAT2 or FAT4 gene was not identified by using the BLAST programs. Based on these facts, it was concluded FAT1-MTNR1A and FAT3-MTNR1B loci were paralogous regions within the human genome (Fig. 2B).

Full-length complete CDS of human FAT1, FAT2 and FAT3 (Fig. 2A) were used as query sequences for the BLAST programs to identify genome clones corresponding to FAT family genes. The 5'-flanking promoter regions of human FAT1, FAT2 and FAT3 genes were identified within AC107050.3, AC011374.6 and AP003124.3 genome sequences, respectively. Repetitive sequence was located within the 5'-promoter region of FAT3 gene. TCF/LEF-binding sites within the 5'-promoter region of FAT1, FAT2 and FAT3 genes were then searched for based on manual inspection. One TCF/LEF-binding site was located about 1100 bp upstream of the transcription start site of FAT1 gene, and also about 800 bp upstream of the transcription start site of the FAT2 gene.

Comparative proteomics analyses on the FAT family. FAT1, FAT2, FAT3 and FAT4 are type I transmembrane proteins. Extracellular region of FAT1 and FAT3 consisted of 33 Cadherin repeats, one Laminin $\mathrm{G}$ (LamG) domain and two EGF domains. Extracellular region of FAT2 consisted of 32 Cadherin repeats, one LamG domain and two EGF domains. Extracellular region of FAT4 consisted of 34 Cadherin repeats, two LamG domains and three EGF domains. Phylogenetic analyses revealed that FAT1, FAT2 and FAR3 were divergent from FAT4 (Fig. 2C).

Although extracellular region was relatively well conserved among FAT family members, cytoplasmic region was divergent. Based on the alignment of C-terminal part of FAT family members, cytoplasmic VCSVxPxLP and SDYxS motifs were identified as novel motifs conserved among FAT1, FAT2 and FAT3 orthologs (Fig. 2D).

Expression profile of human FAT family members. In silico expression analyses were performed to investigate the 
A

\begin{tabular}{|c|c|c|c|c|c|c|c|}
\hline $\begin{array}{c}\begin{array}{c}\text { Exon } \\
\text { No }\end{array} \\
\begin{array}{l}01 \\
02\end{array}\end{array}$ & \multicolumn{3}{|c|}{$\begin{array}{l}\text { Nucleotide sequence of human FAT3 } \\
\text { gene around exon - Intron boundaries }\end{array}$} & \multirow{2}{*}{$\begin{array}{c}\text { Exon } \\
\text { No }\end{array}$} & \multicolumn{3}{|c|}{$\begin{array}{l}\text { Nucleotide sequence of human FAT3 } \\
\text { gene around exon - intron boundaries }\end{array}$} \\
\hline $\begin{array}{l}01 \\
02 \\
03 \\
03 \\
04 \\
05 \\
06 \\
07 \\
07 \\
08 \\
09 \\
10 \\
11 \\
12 \\
13\end{array}$ & 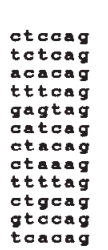 & 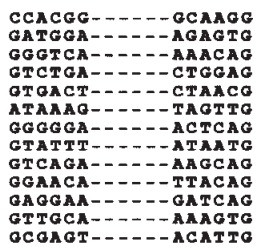 & $\begin{array}{l}\text { gtgogt } \\
\text { gtangt } \\
\text { gtaagg } \\
\text { gtange } \\
\text { gtanga } \\
\text { gtaagt } \\
\text { gtgaga } \\
\text { gtagga } \\
\text { gtgaga } \\
\text { gtgagt } \\
\text { gtgaga } \\
\text { gtaaga } \\
\text { gtangt }\end{array}$ & & 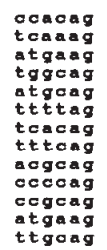 & 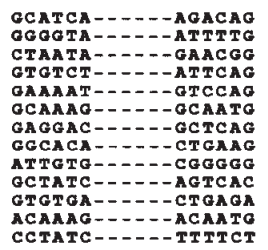 & 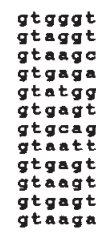 \\
\hline
\end{tabular}

B

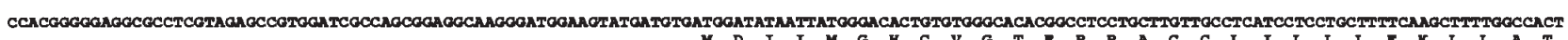

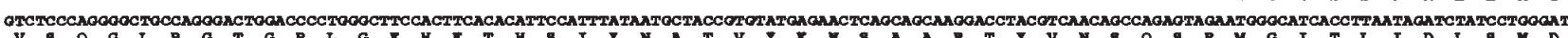

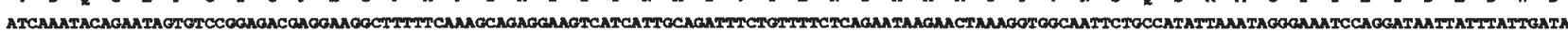
$R$ I R T X G G M 8 T

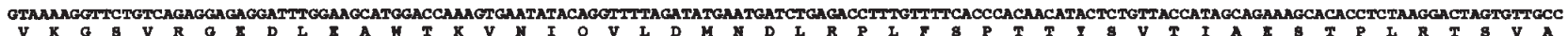

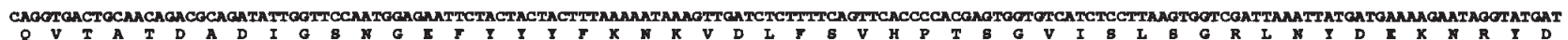

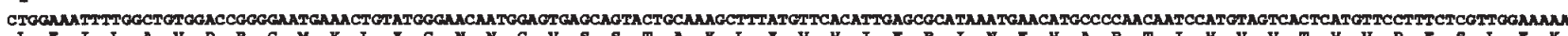

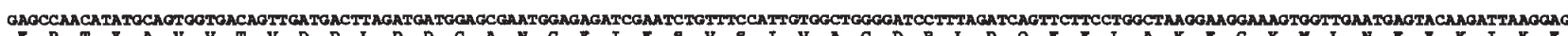

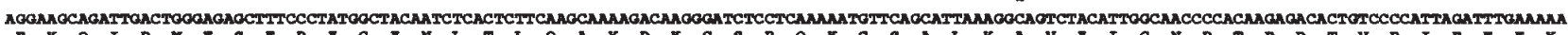

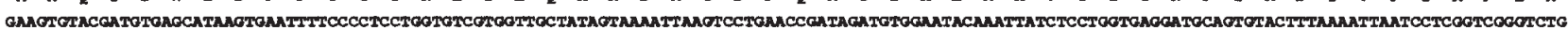

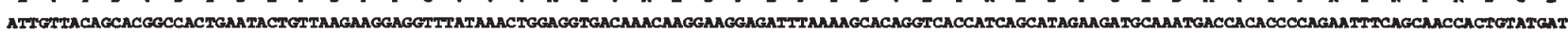

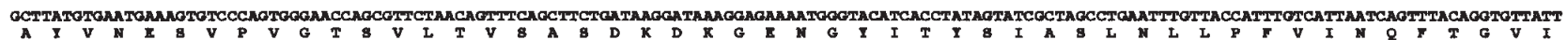

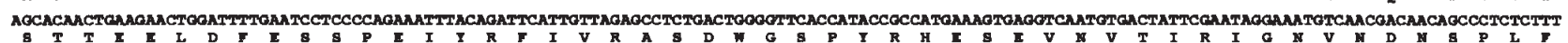

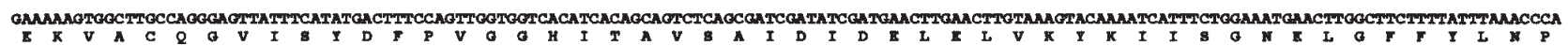

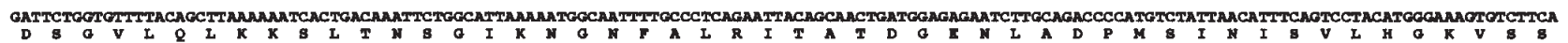

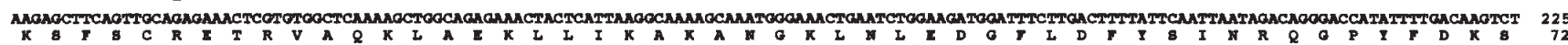

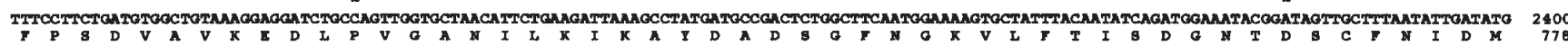

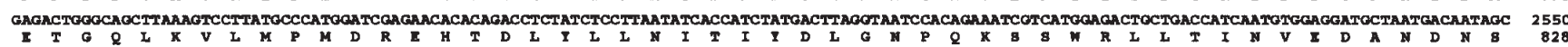

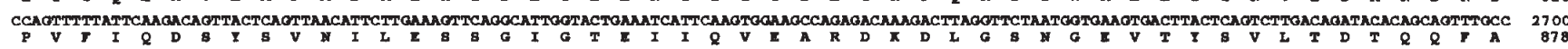

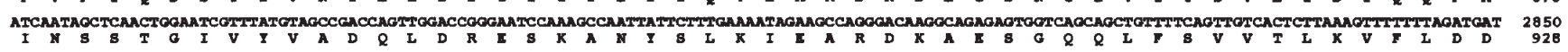

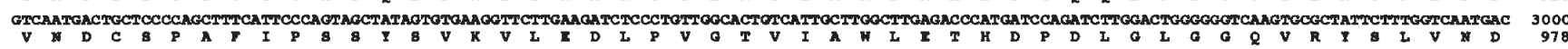

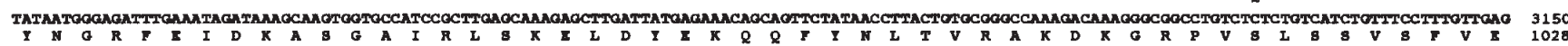

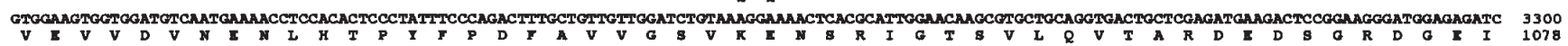

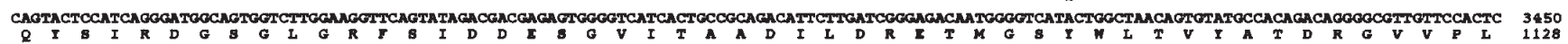

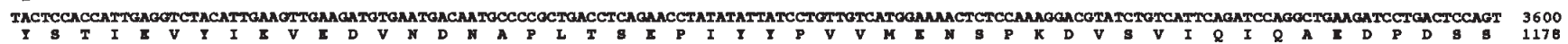

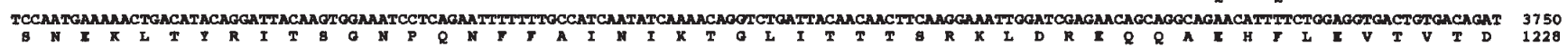

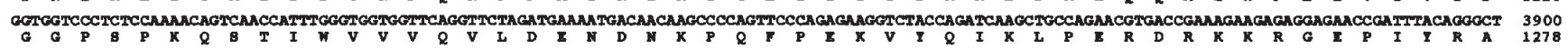

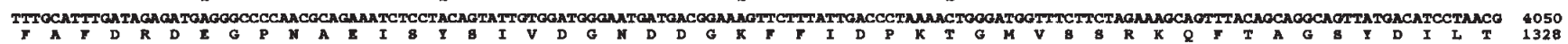

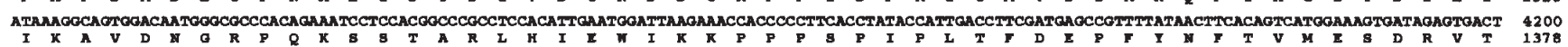

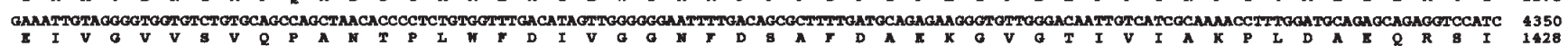

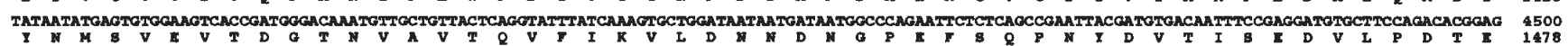

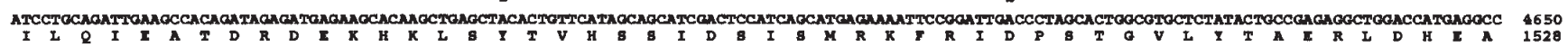

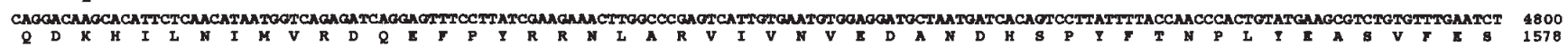

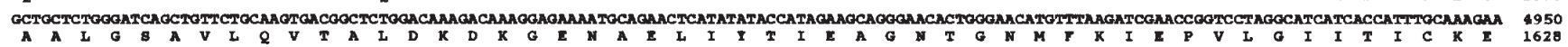

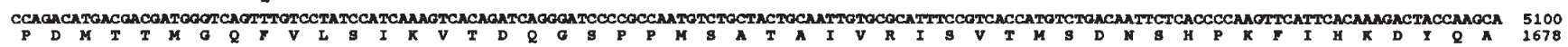

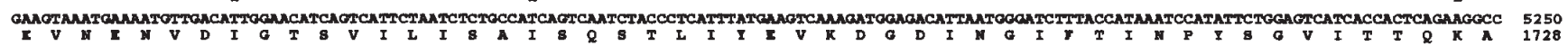

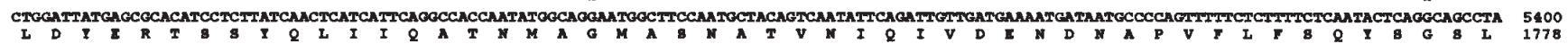

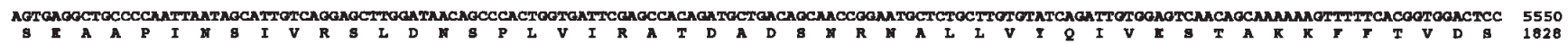

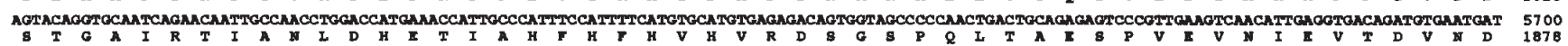

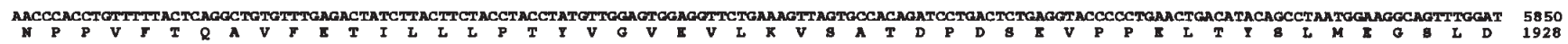

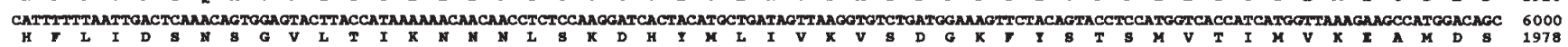

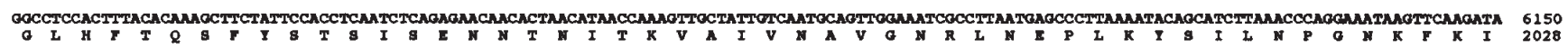

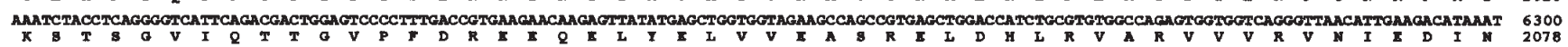

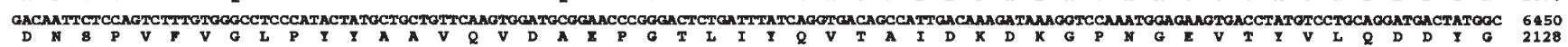

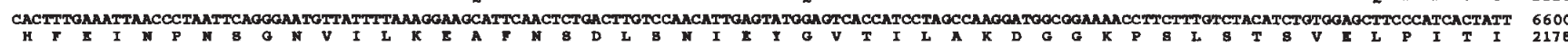




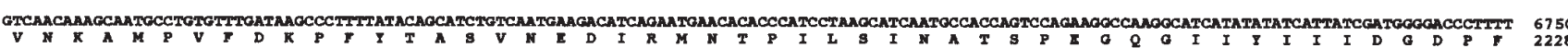

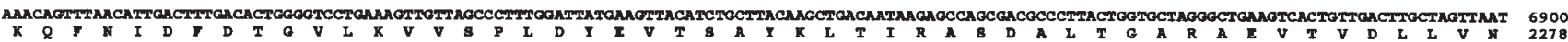

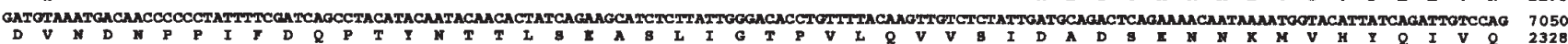

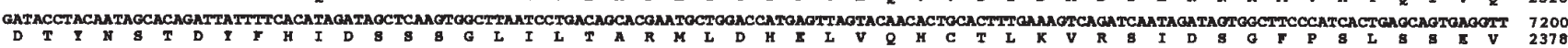

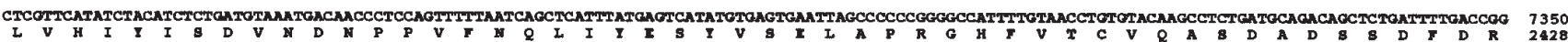

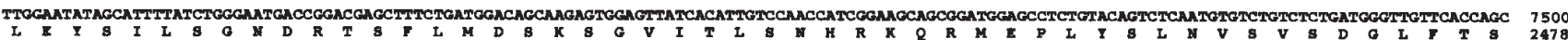

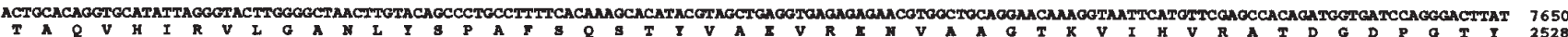

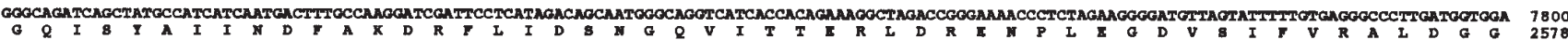

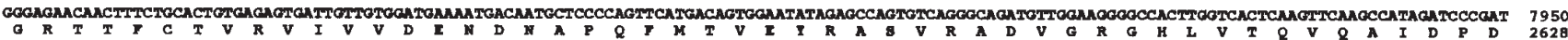

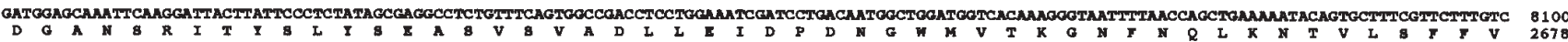

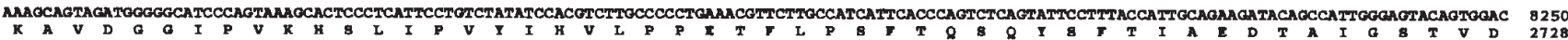

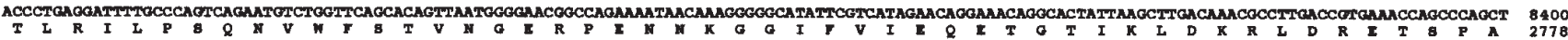

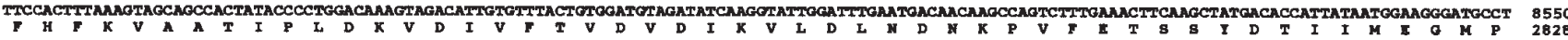

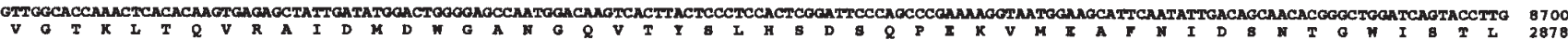

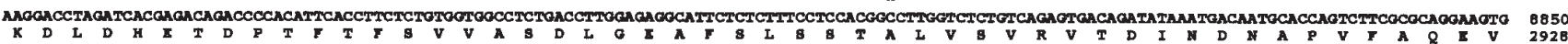

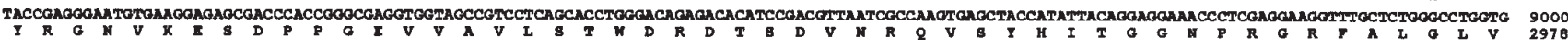

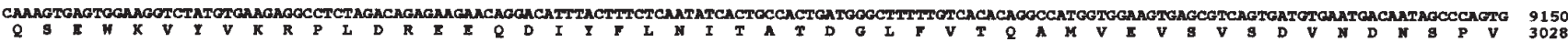

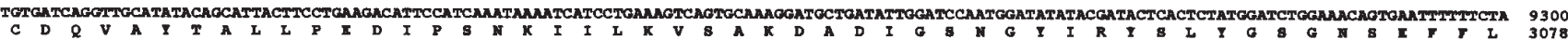

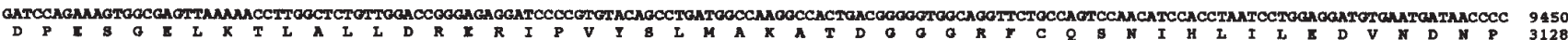

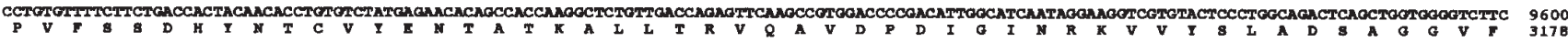

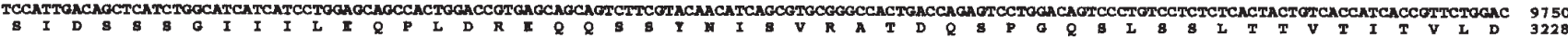

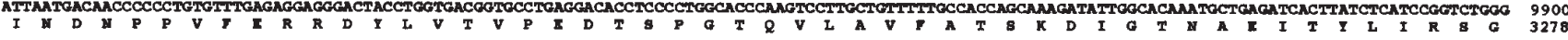

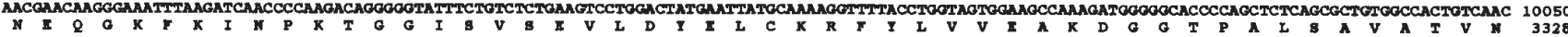

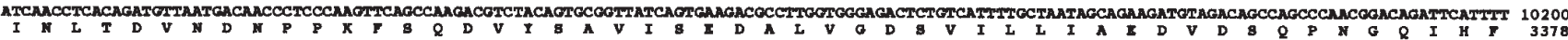

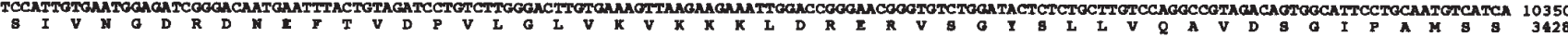

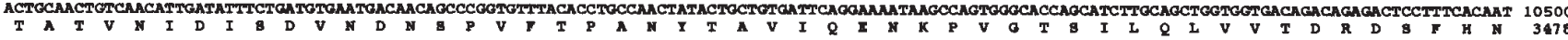

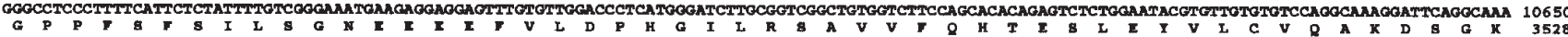

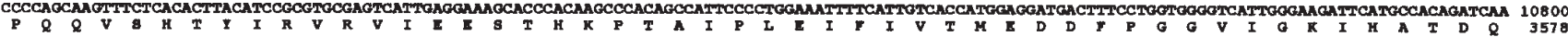

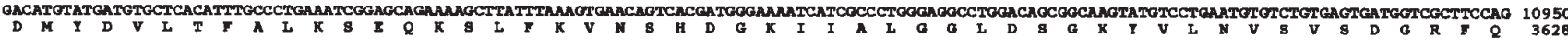

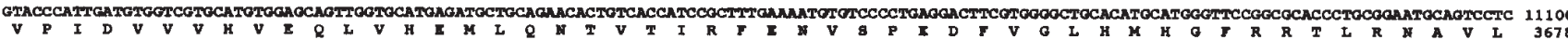

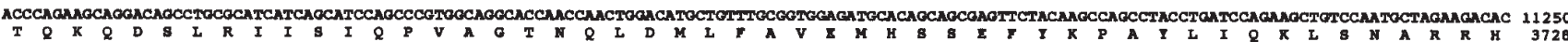

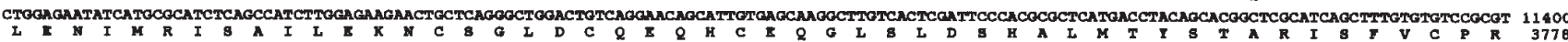

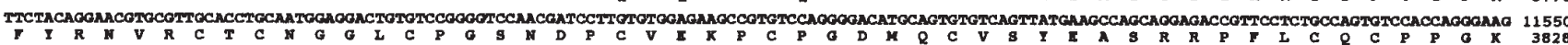

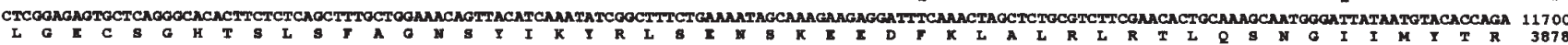

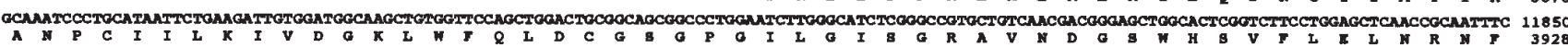

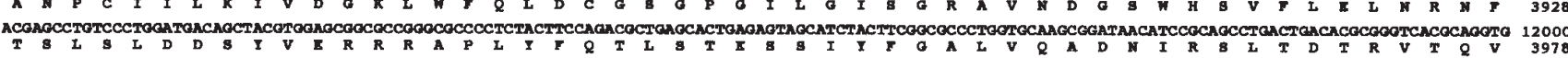

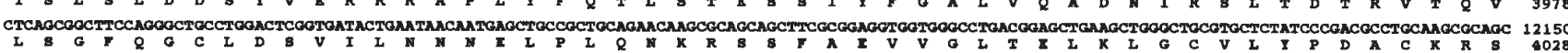

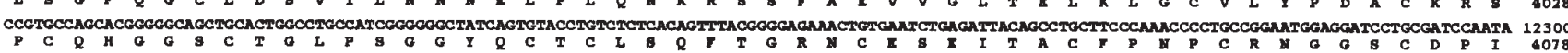

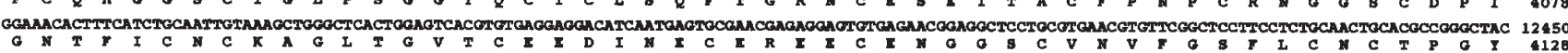

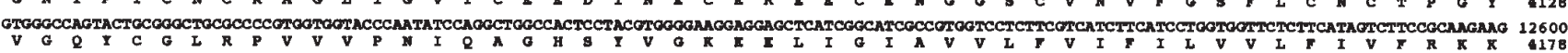

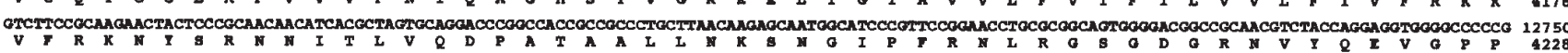

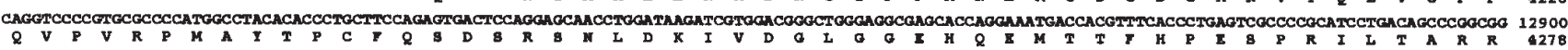

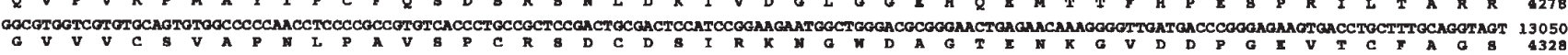

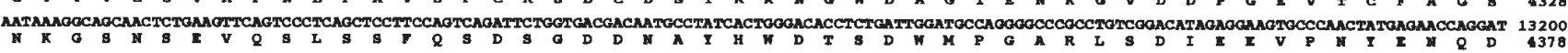

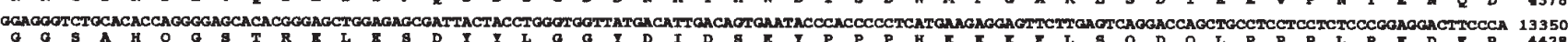

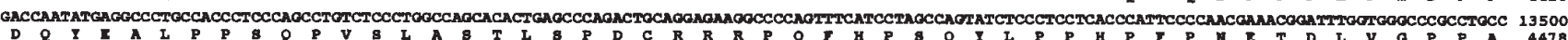

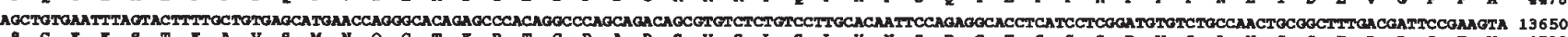

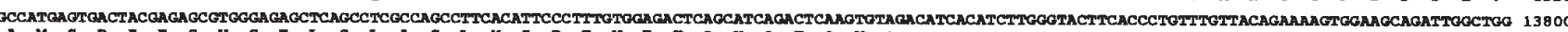

Figure 1. Human FAT3. (A), Exon-intron structure of human FAT3 gene. (B), Nucleotide and amino-acid sequences of human FAT3 complete CDS. Nucleotides and amino-acid residues are numbered on the right.

expression profile of human FAT1, FAT2, FAT3 and FAT4 mRNAs. FAT1 mRNA was expressed in embryonic stem (ES) cells, neural tissues, and also in a variety of tumors, such as gastric cancer, pancreatic cancer, colorectal cancer, breast cancer, lung cancer and brain tumors. FAT2 mRNA was expressed in infant brain, cerebellum, and also in a variety of tumors, such as pancreatic cancer, diffuse type gastric cancer, ovarian cancer, esophageal cancer, skin squamous cell 
A

\begin{tabular}{|c|c|c|c|}
\hline $\begin{array}{c}\text { Human } \\
\text { gene }\end{array}$ & Aliao & $\begin{array}{l}\text { Chromosomad } \\
\text { localizanion }\end{array}$ & Full -length complete CDS \\
\hline FATI & COHFY & 4935.2 & HM_005245.3 \\
\hline FATR & COHFO & $5 q 33.1$ & A00embly of DA102144.1 and AM_001447.1 \\
\hline FATS & COHFIS & $11 q 14.3-q 21$ & Figure 18 \\
\hline FAT4 & CDHFIA & 4928.1 & Aovembly of $A C 079835.5$ and AM_O$_{2} 024582.2$ \\
\hline
\end{tabular}

C

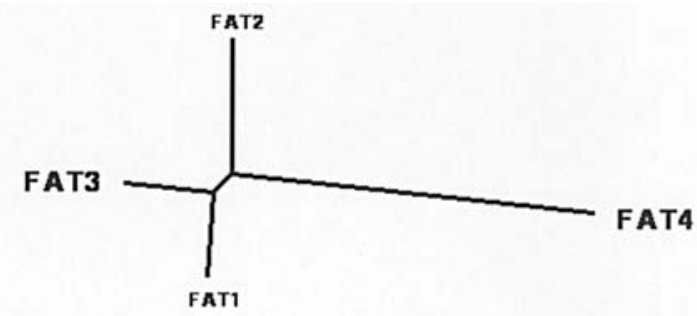

B

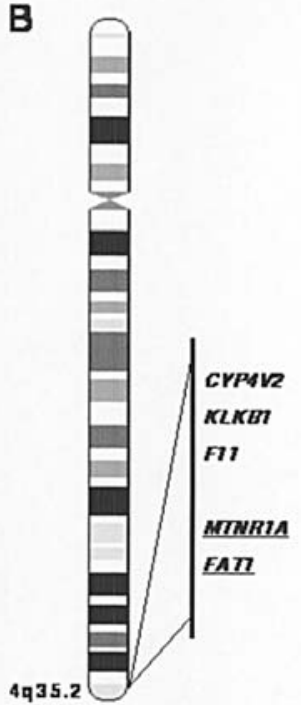

D

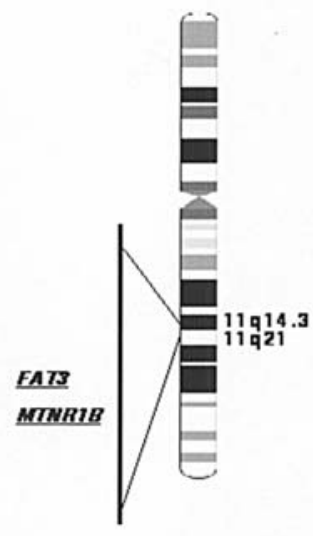

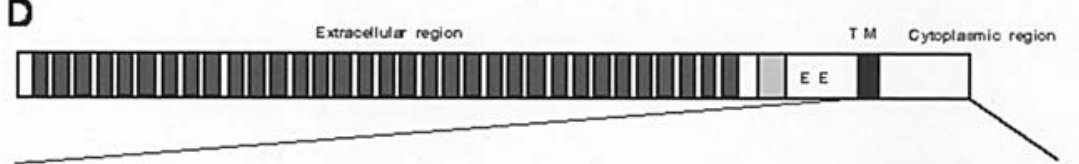

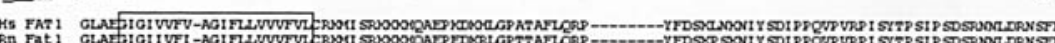

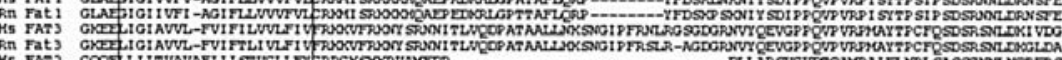

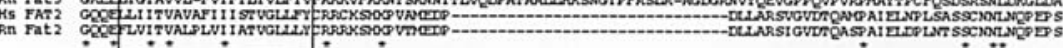

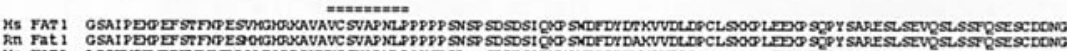
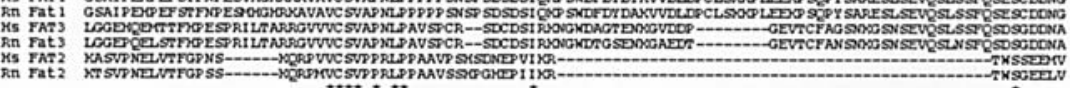

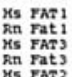

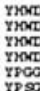

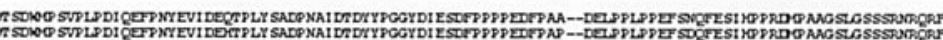

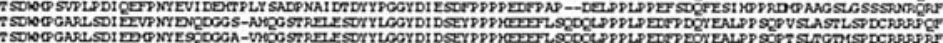

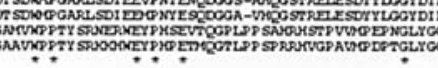

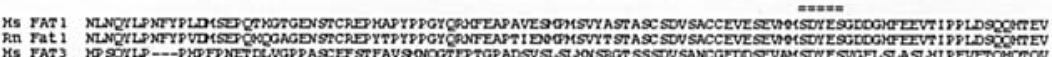

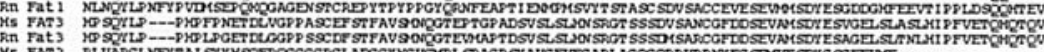

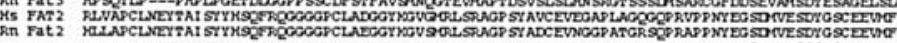

Figure 2. Comparative integromics on FAT family. (A), Human $F A T$ gene family. Gene symbol, alias, chromosomal localization and full-length complete coding sequence of FAT family genes are listed. (B), Intra-species comparative genomics on FAT1 and FAT3 loci. FAT1-MTNR1A locus at 4q35.2 and FAT3-MTNR1B locus at 11q14.3-q21 are paralogous regions within the human genome. (C), Phylogenetic analysis on human FAT family members. FAT1, FAT2 and FAT3 are divergent from FAT4. (D), Schematic representation of FAT3 and partial alignment of FAT1, FAT2 and FAT3 orthologs. Extracellular region, consisting of Cadherin repeats (dark gray box), Laminin G domain (light gray box) and EGF repeat (E), is well conserved among FAT family members; however cytoplasmic region is divergent. The regions around transmembrane domain and cytoplasmic region of FAT1, FAT2 and FAT3 orthologs are aligned. Hs, human; Rn, rat. Conserved amino-acid residues are shown by asterisks. Two novel cytoplasmic motifs VCSVxPxLP and SDYxS are shown by double overlines.

carcinoma, head and neck cancer. FAT3 mRNA was expressed in ES cells, primitive neuroectoderm, fetal brain, infant brain, adult neural tissues and prostate. FAT4 mRNA was expressed in fetal brain, infant brain, brain tumor and colorectal cancer.

\section{Discussion}

Complete CDS of human FAT3 and FAT4 were determined for the comparative integromic analyses on FAT family members in this study. FAT3 gene, consisting of 26 exons, was found to encode a 4557 -aa protein with extracellular 33 Cadherin repeats, one LamG domain and two EGF domains (Fig. 1). FAT4 gene was found to encode a 4924-aa protein with extracellular 34 Cadherin repeats, two LamG domains and three EGF domains. Extracellular region of FAT family members with Cadherin repeats, LamG domain and EGF domains was relatively well conserved; however, cytoplasmic region was divergent. Cytoplasmic VCSVxPxLP and SDYXS motifs were identified as novel motifs conserved among FAT1, FAT2 and FAT3 orthologs (Fig. 2D). Domain architecture comparison and phylogenetic analysis revealed that FAT1, FAT2 and FAR3 were divergent from FAT4.

Intra-species comparative genomics revealed that FAT1-MTNRIA locus at 4q35.2 and FAT3-MTNRIB locus at 11q14.3-q21 were paralogous regions within the human genome (Fig. 2B). Comparative proteomics analyses revealed that C-terminal PDZ-binding motif was conserved among
FAT1 and FAT3 orthologs. Together, these facts indicate that FAT1 and FAT3 are paralogs.

FAT1 mRNA was expressed in ES cells, neural tissues, and a variety of tumors. FAT2 mRNA was expressed in infant brain, cerebellum, and a variety of tumors. FAT3 mRNA was expressed in ES cells, primitive neuroectoderm, fetal brain, infant brain, adult neural tissues, and prostate. FAT4 mRNA was expressed in fetal brain, infant brain, brain tumor and colorectal cancer. FAT family members are implicated in the early embryogenesis and neurogenesis.

The canonical WNT signaling pathway cross-talks with FGF, Notch, Hedgehog and BMP signaling pathways during embryogenesis, chronic persistent inflammation and carcinogenesis (49-56). Because canonical WNT signaling activation leads to transcriptional activation of target genes through the TCF/LEF, B-catenin, Legless and Pygo complex, TCF/LEFbinding site within the promoter region of human FAT family genes were investigated. A single TCF/LEF-binding site was identified within FAT1 and FAT2 promoters; however, the relationship between the canonical WNT signaling activation and the expression of FAT1 or FAT2 in a variety of human tumors remains to be elucidated.

Drosophila Fat functions as a tumor suppressor (27). Tumor suppressor gene is inactivated due to epigenetic change (CpG hypermethylation) and genetic alterations (mutation or deletion) during multi-stage carcinogenesis $(57,58)$. Epigenetic changes and genetic alterations of FAT family genes in a 
variety of human tumors should be investigated in the future. FAT family members were revealed to be targets of systems medicine in the fields of oncology and neurology.

\section{References}

1. Adler PN: Planar signaling and morphogenesis in Drosophila. Dev Cell 2: 525-535, 2002.

2. Vinson CR, Conover S and Adler PN: A Drosophila tissue polarity locus encodes a protein containing seven potential transmembrane domains. Nature 338: 263-264, 1989.

3. Wolff T and Rubin GM: Strabismus, a novel gene that regulates tissue polarity and cell fate decisions in Drosophila. Development 125: 1149-1159, 1998.

4. Heitzler P, Coulson D, Saenz-Robles MT, et al: Genetic and cytogenetic analysis of the 43A-E region containing the segment polarity gene costa and the cellular polarity genes prickle and spiny-legs in Drosophila melanogaster. Genetics 135: 105-115, 1993.

5. Chae J, Kim MJ, Goo JH, et al: The Drosophila tissue polarity gene starry night encodes a member of the protocadherin family. Development 126: 5421-5429, 1999.

6. Feiguin F, Hannus M, Mlodzik M and Eaton S: The ankyrin repeat protein Diego mediates Frizzled-dependent planar polarization. Dev Cell 1: 93-101, 2001.

7. Katoh M: WNT/PCP signaling pathway and human cancer. Oncol Rep 14: 1583-1588, 2005.

8. Saitoh T and Katoh M: Frequent up-regulation of WNT5A mRNA in primary gastric cancer. Int J Mol Med 9: 515-519, 2002.

9. Saitoh T and Katoh M: Molecular cloning and characterization of human $W N T 5 B$ on chromosome 12 p13.3 region. Int J Oncol 19: 347-351, 2001

10. Katoh $\mathrm{M}$ and Katoh M: Comparative genomics on WNT5A and WNT5B genes. Int J Mol Med 15: 749-753, 2005.

11. Kirikoshi H, Sekihara H and Katoh M: Molecular cloning and characterization of human WNT11. Int J Mol Med 8: 651-656, 2001.

12. Katoh Y and Katoh M: Comparative genomics on WNT11 gene. Int J Mol Med 15: 879-883, 2005.

13. Heller RS, Klein T, Ling Z, Heimberg H, Katoh M, Madsen OD and Serup P: Expression of WNT, Frizzled, SFRP and DKK genes in adult human pancreas. Gene Expr 11: 141-147, 2003.

14. Kirikoshi H, Koike J, Sagara N, Saitoh T, Tokuhara M, Tanaka K, Sekihara H, Hirai $\mathrm{M}$ and Katoh M: Molecular cloning and genomic structure of human Frizzled-3 at chromosome 8p21. Biochem Biophys Res Commun 271: 8-14, 2000.

15. Kirikoshi H, Sekihara $\mathrm{H}$ and Katoh M: Expression profiles of 10 members of Frizzled gene family in human gastric cancer. Int $\mathbf{J}$ Oncol 19: 767-771, 2001

16. Masiakowski P and Carroll RD: A novel family of cell surface receptors with tyrosine kinase-like domain. J Biol Chem 267: 26181-26190, 1992.

17. Katoh $\mathrm{M}$ and Katoh M: Identification and characterization of rat Ror 1 and Ror 2 genes in silico. Int J Mol Med 15: 533-538, 2005.

18. Katoh $\mathrm{M}$ and Katoh M: Comparative genomics on RORI and ROR2 orthologs. Oncol Rep 14: 1381-1384, 2005.

19. Katoh M: Molecular cloning and characterization of Strabismus 2 (STB2). Int J Oncol 20: 993-998, 2002.

20. Katoh M: Structure and expression of Strabismus 1 (STB1) gene on human chromosome 1q21-q23. Int J Oncol 20: 1197-1203, 2002.

21. Katoh M: Strabismus (STB)/Vang-like (Vangl) gene family (Review). Int J Mol Med 10: 11-15, 2002.

22. Katoh Y and Katoh M: Comparative genomics on VANGL1 and VANGL2 genes. Int J Oncol 26: 1435-1440, 2005.

23. Katoh $M$ and Katoh $M$ : Identification and characterization of human PRICKLE1 and PRICKLE2 genes as well as mouse Prickle1 and Prickle2 genes homologous to Drosophila tissue polarity gene prickle. Int J Mol Med 11: 249-256, 2003.

24. Katoh M and Katoh M: Identification and characterization of rat Ankrd6 gene in silico. Int J Mol Med 15: 359-363, 2005.

25. Lu X, Borchers AG, Jolicoeur C, et al: PTK7/CCK-4 is a novel regulator of planar cell polarity in vertebrates. Nature 430: 93-98, 2004.

26. Wu Q and Maniatis T: A striking organization of a large family of human neural cadherin-like cell adhesion genes. Cell 97: 779-790, 1999.
27. Bryant PJ, Huettner B, Held LL Jr, Ryerse J and Szidonya J: Mutations at the fat locus interfere with cell proliferation control and epithelial morphogenesis in Drosophila. Dev Biol 129: 541-554, 1988.

28. Cho E and Irvine KD: Action of fat, four-jointed, dachsous and dachs in distal-to-proximal wing signaling. Development 131: 4489-4500, 2004.

29. Fanto $\mathrm{M}$ and McNail $\mathrm{H}$ : Planar cell polarity from fly to vertebrates. J Cell Sci 117: 527-533, 2004.

30. Castillejo-Lopez C, Arias WM and Baumgartner S: The fat-like gene of Drosophila is the true ortholog of vertebrate fat cadherins and is involved in the formation of tubular organs. J Biol Chem 279: 24034-24043, 2004.

31. Ponassi M, Jacques TS, Ciani L and French Constant C: Expression of the rat homologue of the Drosophila fat tumour suppressor gene. Mech Dev 80: 207-212, 1999.

32. Rock R, Schrauth S and Gessler M: Expression of mouse dchs1, fjx 1 and fat-j suggests conservation of the planar cell polarity pathway identified in Drosophila. Dev Dyn 234: 747-755, 2005.

33. Tanoue T and Takeichi M: New insights into Fat cadherins. J Cell Sci 118: 2347-2353, 2005.

34. Katoh M: Paradigm shift in gene-finding method: from bench-top approach to desk-top approach. Int J Mol Med 10: 677-682, 2002.

35. Katoh $M$ and Katoh $M$ : Identification and characterization of human HES2, HES3 and HES5 genes in silico. Int J Oncol 25: 529-534, 2004.

36. Katoh $\mathrm{M}$ and Katoh $\mathrm{M}$ : Identification and characterization of human HESL, rat Hesl and rainbow trout hesl genes in silico. Int J Mol Med 14: 747-751, 2004.

37. Katoh $\mathrm{Y}$ and Katoh M: Identification and characterization of rat Wnt1 and Wnt10b genes in silico. Int J Oncol 26: 841-845, 2005.

38. Katoh $\mathrm{M}$ and Katoh $\mathrm{M}$ : Comparative genomics on WNT8A and WNT8B genes. Int J Oncol 26: 1129-1133, 2005.

39. Katoh M: Comparative genomics on WNT3-WNT9B gene cluster. Int J Mol Med 15: 743-747, 2005.

40. Katoh Y and Katoh M: Comparative genomics on DKK1 orthologs. Int J Oncol 27: 275-279, 2005.

41. Katoh Y and Katoh M: Comparative genomics on $D K K 2$ and DKK4 orthologs. Int J Mol Med 16: 477-481, 2005.

42. Katoh Y and Katoh M: Comparative genomics on FGF 16 orthologs. Int J Mol Med 16: 959-963, 2005.

43. Katoh $\mathrm{M}$ and Katoh M: Comparative genomics on FGF8, FGF17 and FGF18 orthologs. Int J Mol Med 16: 493-496, 2005.

44. Katoh M: Molecular evolution of WNT2B orthologs. Int J Oncol 26: 1135-1139, 2005.

45. Katoh Y and Katoh M: Comparative genomics on SFRPI orthologs. Int J Oncol 27: 861-865, 2005.

46. Katoh Y and Katoh M: Comparative genomics on HHIP family orthologs. Int J Mol Med 17: 391-395, 2006.

47. Katoh Y and Katoh M: FGF signaling inhibitor, SPRY4, is evolutionarily conserved target of WNT signaling pathway in progenitor cells. Int J Mol Med 17: 529-532, 2006.

48. Katoh M and Katoh M: Notch ligand, JAG1, is evolutionarily conserved target of canonical WNT signaling pathway in progenitor cells. Int J Mol Med 17: 681-685, 2006.

49. Katoh M: $W N T$ and $F G F$ gene clusters. Int J Oncol 21: 1269-1273, 2002.

50. Katoh $\mathrm{M}$ and Katoh $\mathrm{M}$ : Identification and characterization of human $B C L 9 L$ gene and mouse Bcl9l gene in silico. Int J Mol Med 12: 643-649, 2003

51. Garciadiego-Cazares D, Rosales C, Katoh M and ChimalMonroy J: Coordination of chondrocyte differentiation and joint formation by $\alpha 5 \beta 1$ integrin in the developing appendicular skeleton. Development 131: 4735-4742, 2004.

52. Katoh M: Epithelial-mesenchymal transition in gastric cancer. Int J Oncol 27: 1677-1683, 2005.

53. Katoh M: WNT2B: Comparative integromics and clinical application. Int J Mol Med 16: 1103-1108, 2005.

54. Swain RK, Katoh M, Medina A and Steinbeisser H: Xenopus frizzled-4S, a splicing variant of Xfz4, is a context-dependent activator and inhibitor of Wnt/ß-catenin signaling. Cell Commun Signal 3: 12, 2005.

55. Katoh Y and Katoh M: Hedgehog signaling pathway and gastric cancer. Cancer Biol Ther 4: 1045-1049, 2005.

56. Katoh Y and Katoh M: WNT antagonist, SFRP1, is Hedgehog signaling target. Int J Mol Med 17: 171-175, 2006.

57. Katoh M and Katoh M: Pharmacogenomics on gastric cancer. Cancer Biol Ther 3: 566-567, 2004.

58. Katoh $\mathrm{M}$ and Katoh M: Bioinformatics for cancer management in the post-genome era. Technol Cancer Res Treat 5: 169-176, 2006. 